\title{
Berlin, « épicentre » de la vie juive en Allemagne
} (1871-1933)

Berlin, „Epizentrum“ jüdischer Lebenswelten in Deutschland (1871-1933)

Berlin, 'Epicenter' of Jewish Life in Germany (1871-1933)

\section{Laurence Guillon}

\section{OpenEdition}

Journals

Édition électronique

URL : https://journals.openedition.org/ceg/7032

DOI : $10.4000 /$ ceg. 7032

ISSN : 2605-8359

\section{Éditeur}

Presses Universitaires de Provence

\section{Édition imprimée}

Date de publication : 25 septembre 2019

Pagination : $53-74$

ISBN : 979-10-320-0234-6

ISSN : 0751-4239

Référence électronique

Laurence Guillon, «Berlin, «épicentre » de la vie juive en Allemagne (1871-1933) », Cahiers d'Études Germaniques [En ligne], 77 | 2019, mis en ligne le 25 mars 2021, consulté le 15 juin 2021. URL : http:// journals.openedition.org/ceg/7032; DOI : https://doi.org/10.4000/ceg.7032 


\title{
Berlin, « épicentre » de la vie juive en Allemagne (1871-1933)
}

\author{
Laurence GUILLON \\ Université Lumière-Lyon 2 / LCE (EA 1853)
}

\begin{abstract}
«Une histoire d'amour et une tragédie » : c'est en ces termes que Dominique Bourel qualifie la relation fondamentalement ambiguë des Juifs à Berlin ${ }^{1}$, symbolisée par le destin de Walther Rathenau, qui considérait sa ville de naissance comme la plus belle ville du monde et qui y fut pourtant assassiné alors qu'il avait atteint le sommet de sa carrière politique ${ }^{2}$. La capitale du nouvel Empire allemand est une ville récente, comme nous le rappelle Ludwig Geiger, auteur d'une Histoire des Juifs de Berlin en 1871, document-témoin de l'intégration réussie des Juifs dans la société bourgeoise ${ }^{3}$ : comme l'Empire nouvellement créé, elle est animée d'un nouvel élan, d'une atmosphère de créativité particulière, qui n'est sans doute pas étrangère à la réussite spectaculaire des Juifs berlinois, ni à la haine profonde qui s'y est développée à leur égard. Sous l'Empire et jusqu'à la fin de la République de Weimar, Berlin s'affirme de plus en plus comme l'« épicentre ${ }^{4}$ » de la vie juive en Allemagne, l'endroit où celle-ci fut la plus intense et la plus variée, même s'il n'en offre pas exactement une image réduite ou condensée. Au contraire, la vie juive y a ses spécificités, liées bien sûr au caractère urbain de Berlin, à son statut de capitale d'un nouvel État en pleine expansion, à sa transformation en métropole d'échelle mondiale, mais aussi à son histoire et à sa géographie particulières, bref à sa « logique propre ${ }^{5}$ ». Y aurait-il donc un esprit de tolérance et d'ouverture spécifique à la ville, à cette ville en particulier, une ville « toujours en
\end{abstract}

1. Dominique Bourel, «Berlin et les juifs : Une histoire d'amour et une tragédie » in Étienne François, Egon Graf Westerholt (dir.), Berlin : Capitale, mythe, enjeu, Nancy, Presses Universitaires de Nancy, 1988 , p. 87.

2. Nommé ministre des Affaires étrangères le 31 janvier 1922 (la plus haute fonction qu'un homme juif ait jamais exercée en Allemagne), il est assassiné le 22 juin de la même année.

3. Ludwig Geiger, Geschichte der Juden in Berlin, Berlin, Verlag Guttentag, 1871, p. V : « Die Stadt ist neu wie der ganze Staat. »

4. L'expression est empruntée à Jacques Ehrenfreund : Mémoire juive et nationalité allemande. Les juifs berlinois à la Belle Époque, Paris, PUF, 2000, p. 44. Le terme est d'ailleurs forgé par un scientifique allemand en 1874, Johann Friedrich Julius Schmidt dans son ouvrage Studien über Vulkane und Erdbeben.

5. Voir Helmuth Berking, Martina Löw, Die Eigenlogik der Städte: Neue Wege für die Stadtforschung, Frankfurt a. M., Campus, 2008. 
devenir ${ }^{6}$ ", dont l'une des caractéristiques premières serait précisément l'absence de traditions ${ }^{7}$, offrant ainsi plus de possibilités de développement aux vies juives que n'importe quel autre cadre?

Au départ, rien ne prédisposait Berlin à devenir l'épicentre de la vie juive en Allemagne, puisque la présence juive à Berlin ne remonte qu'au XIII ${ }^{\mathrm{e}}$ siècle, alors que la région du Rhin est peuplée de Juifs dès le IV ${ }^{e}$ siècle après J.C. Coïncidence troublante, le premier témoignage de vie juive est une pierre tombale retrouvée à l'emplacement de l'ancien cimetière de Spandau (Judenkiewer) datée de 1244 ; or 1244 est également l'année de la première source écrite mentionnant la ville de Berlin. Ainsi, pour Reinhard Rürup, I'histoire de Berlin et l'histoire des Juifs à Berlin sont «étroitement imbriquées depuis le commencement ${ }^{8}$ ». Cependant, en raison des différentes vagues de persécution des Juifs, on ne peut véritablement parler d'une continuité de la vie juive à Berlin qu'à partir de 1671, lors de l'arrivée de cinquante familles chassées de Vienne et accueillies par le " grand " princeélecteur Frédéric-Guillaume, deux cents ans tout juste avant le début de notre période d'étude. L'ascension a donc été fulgurante. Quoi qu'il en soit, si cette vie juive ou ces différentes formes de vie juive à Berlin présentent une caractéristique, c'est précisément la diversité (et même une diversification croissante), comme l'a très bien rappelé l'événement « Zerstörte Vielfalt » en 2013, qui a mis en lumière la perte incommensurable infligée à la ville de Berlin par la perte de sa communauté juive ${ }^{9}$.

Si nous avons choisi une démarche thématique, en considérant la vie juive à Berlin d'abord sous l'angle de sa vitalité (démographique, économique et sociale, ainsi que religieuse), puis de sa créativité, et enfin dans la perspective des menaces qui planent sur elle, rappelons qu'une césure est néanmoins déterminante pour notre propos : la réforme administrative du « Grand Berlin » en 1920, qui fait de la capitale allemande la plus grande ville du monde en termes de superficie $\left(890 \mathrm{~km}^{2}\right)$ et la troisième en termes de population avec près de quatre millions d'habitants ${ }^{10}$.

6. Karl Scheffler, Berlin. Ein Stadtschicksal, éd. par Florian Illies, Berlin, Suhrkamp, 2015 [1910] : "Berlin ist dazu verdammt, immerfort zu werden und niemals zu sein. »

7. Voir Joachim Schlör, Das Ich der Stadt. Debatten über Judentum und Urbanität, 1822-1938, Göttingen, Vandenhoeck \& Ruprecht, 2005.

8. Reinhard Rürup (dir.), Jüdische Geschichte in Berlin. Bilder und Dokumente, Berlin, Hentrich, 1995, p. 6.

9. Cet ensemble de manifestations s'est déroulé dans toute la ville de Berlin à l'occasion des 80 ans de l'arrivée au pouvoir de Hitler et des 75 ans de la « Nuit de Cristal ».

10. Berlin avait déjà connu un premier agrandissement d'ampleur en 1861, puis les murs d'enceinte furent rasés en 1867-1868; enfin, trois territoires y furent rattachés en 1878, 1881 et 1915 avant la grande réforme de 1920 . 


\section{Une communauté juive d'une vitalité exceptionnelle}

\section{Essor démographique}

La communauté juive allemande se caractérise dans son ensemble par un phénomène d'urbanisation massive, qui commence vers le milieu du XIXe siècle et prend son essor à partir de 1871, au moment où Berlin devient capitale. Alors qu'en 1813, moins de 3000 Juifs vivaient à Berlin, et 10000 en 1850, ils étaient 36000 en 1871, ce qui représentait $7 \%$ de l'ensemble de la communauté juive allemande. Le seuil des 100000 membres est atteint au tournant du siècle et en 1925, l'apogée est atteint avec 175000 personnes, soit près d'un tiers de l'ensemble des Juifs allemands. Le chiffre décline avant l'arrivée au pouvoir des nazis, puisqu'on compte 160000 membres en $1933^{11}$.

On voit donc que la population juive de Berlin a été multipliée par quatre au cours de la période d'étude, et que Berlin compte en moyenne $4 \%$ de Juifs, alors qu'on se situe plutôt autour de $1 \%$ dans le reste de l'Allemagne ${ }^{12}$, ce qui explique sans doute la remarque de l'historien Theodor Mommsen dans sa réponse à Treitschke lors de la « querelle de l'antisémitisme » en 1880, selon laquelle à Berlin, l'élément juif et l'élément allemand "se mêlent de fait plus fortement que nulle part ailleurs ${ }^{13}$ ». On est donc loin du tableau pessimiste du «Berlin stérile » dressé par Felix Theilhaber, chantre du déclin démographique dans son ouvrage Le déclin des Juifs allemands en 1911, qui rendait Berlin responsable de ce déclin présumé ${ }^{14}$.

On observe par ailleurs un changement fondamental dans la hiérarchie des villes les plus peuplées de Juifs : les foyers traditionnels de population juive, comme Breslau ou Prague, déclinent considérablement au profit des deux capitales, Berlin et Vienne. Seule Vienne dépasse encore Berlin avec près de 200000 Juifs en 1925, et une proportion de l'ordre de $8 \%$ de Juifs. Fait intéressant également, la population globale de Berlin ne croît pas plus vite que sa population juive, à l'inverse de la tendance nationale ${ }^{15}$.

D'autre part, Berlin exerce une puissante force d'attraction sur les régions à l'entour, ce qui s'explique par le développement de son potentiel industriel et l'exode rural, mais aussi sur les populations étrangères, en particulier les « Juifs

11. Chiffres de Ulrich Wyrwa, «Das jüdische Berlin der Kaiserzeit », in Arno Herzig, Cay Rademacher, Die Geschichte der Juden in Deutschland, Bonn, BPB, 2008, p. 140, et de Ehrenfreund, Mémoire juive et nationalité allemande, p. 43.

12. La ville de Francfort-sur-le-Main constitue une exception notable, puisque sa population juive représente 6 à $7 \%$ de la population globale.

13. Theodor Mommsen, "Auch ein Wort über unser Judentum », in Reden und Aufsätze, Berlin, Weidmann, 1905, p. 410-414 : « in der deutschen Hauptstadt, wo diese Stämme factisch sich stärker mischen als irgendwo sonst ».

14. Felix Aron Theilhaber, Der Untergang der deutschen Juden. Eine volkswirtschaftliche Studie, München, Reinhardt, 1911, p. 56.

15. Elles s'accroissent au même rythme, la population juive dépassant même le rythme de la population d'ensemble entre 1910 et 1925 (+ $20 \%$ contre $8 \%$ ). Voir Joachim Schlör, « Ankommen in Berlin. Eine Projektskizze », in Laurence Guillon, Heidi Knörzer (dir.), Berlin und die Juden. Geschichte einer Wahlverwandtschaft, Berlin, Neofelis Verlag, 2015, p. 31. 
de l'Est », dont la présence est beaucoup plus marquée que dans d'autres régions allemandes. Ces populations fuient la Russie tsariste en direction de l'Ouest, notamment à partir de 1880, suite à une vague de pogromes. Certains ne font que traverser l'Allemagne, mais d'autres restent à Berlin pour un certain temps, voire s'y établissent durablement, généralement dans le célèbre quartier des granges (Scheunenviertel). En 1925, 25 \% des Juifs de Berlin étaient nés en dehors de la ville ${ }^{16}$, une proportion beaucoup plus élevée que dans le reste de l'Allemagne, d'où le bon mot qui s'est répandu à l'époque : " als echter Berliner natürlich in Breslau geboren ».

Nous avons donc affaire à une communauté numériquement plus forte et plus visible, plus marquée par l'immigration, ce qui la rend aussi plus jeune et dynamique que les autres communautés juives d'Allemagne, mais qui néanmoins partage avec elles des caractéristiques démographiques semblables : recul de la natalité, taux de mariages mixtes de plus en plus élevé (même encore plus élevé que dans le reste de l'Allemagne), tandis que les conversions (elles aussi généralement plus importantes dans les grandes villes) reculent et le nombre de Juifs qui quittent la communauté juive sans se convertir à une autre religion augmente ${ }^{17}$.

\section{Prospérité économique et sociale}

Parallèlement à cet essor démographique, la population juive berlinoise se caractérise par son intégration massive à la bourgeoisie en à peine quelques décennies. En 1871, les Juifs berlinois constituent dans l'ensemble un groupe social aisé, relativement homogène ${ }^{18}$, ce qui n'exclut pas bien sûr l'existence d'une couche de Juifs pauvres à Berlin. Ces Juifs et Juives (car dans de nombreuses familles, l'épouse juive travaillait dans l'entreprise de son mari sans être officiellement employée - pensons à Betty Scholem, la mère de Gershom, qui travaillait dans l'imprimerie de son mari ${ }^{19}$ ) ont véritablement contribué à l'essor économique de la ville. Comme le rappelle Monika Richarz, " c'est [...] à Berlin que l'importance économique et culturelle de la minorité juive dans la constitution d'un centre urbain se manifestait le plus ${ }^{20} »$. Sur le plan culturel, nous le verrons plus loin ; sur le plan économique, on peut citer les entreprises fondées par des Juifs qui ont fait la renommée internationale de Berlin : les grands magasins (Warenhäuser) comme Wertheim à partir de 1885, dont le logo, un W portant le globe terrestre, est devenu un emblème de la ville, Tietz, où est installé le premier escalier roulant d'Allemagne, Alfred Jandorf et son fameux KaDeWe ouvert en 1906, sans oublier

\footnotetext{
16. Ibid.

17. Monika Richarz in Steven M. Lowenstein, Paul Mendes-Flohr et al. (dir.), Deutsch-jüdische Geschichte in der Neuzeit, t. III, Umstrittene Integration 1871-1918, München, Beck, 1997, p. 1338,

18. Ehrenfreund, Mémoire juive et nationalité allemande, p. 45.

19. Voir M. Richarz in Lowenstein, Mendes-Flohr et al. (dir.), Umstrittene Integration, p. 92.

20. Citée par Schlör, Das Ich der Stadt, p. 100.
} 
Salman Schocken ${ }^{21}$. On voit néanmoins dans le cas de ces directeurs de grands magasins que, malgré leur éclatant succès économique, ils restaient considérés comme des "nouveaux riches », des " parvenus ». En 1900 est d'ailleurs institué un impôt spécial sur les grands magasins, moyen indéniable de taxer les Juifs à une époque où ils détenaient pratiquement le monopole du secteur.

La confection est également un secteur d'activité qui fait la réputation de Berlin et où s'illustrent de nombreuses entreprises juives : le "Konfektionsviertel » se situe aux alentours de la Hausvogteiplatz (avec par exemple l'entreprise Grünfeld). Un peu plus au nord, vers la Behrenstraße, se trouve le «Bankenviertel » (avec des noms comme Mendelssohn, Fürstenberg...) ; au Sud de la Leipziger Straße, c'est le «Zeitungsviertel » qui commence : Rudolf Mosse crée un véritable empire symbolisé par le Berliner Tageblatt fondé en 1872, journal de référence dans toute I'Allemagne pour sa grande qualité. Leopold Ullstein et lui éditent les journaux libéraux dominants de l'époque, qui constituent une voix importante susceptible de donner le ton (un ton résolument libéral) en matière de culture et de politique - une tendance qui décline nettement sous la République de Weimar. Enfin, les grandes industries de pointe (chimie, électricité) fondées par des Juifs se trouvent dans le quartier de Moabit : ainsi l'AEG fondée en 1883 par Emil Rathenau, un "Kaiserjude », sera reprise par son fils en 1914. Par ailleurs, la représentation dans les métiers «traditionnellement juifs » est encore plus marquée à Berlin que dans le reste de l'Allemagne : ainsi, au début du XXe siècle, la moitié des médecins de Berlin étaient des Juifs et plus de la moitié des avocats ${ }^{22}$.

Cette ascension sociale se matérialise très concrètement dans la géographie de la ville. Si la plupart des Juifs vivent dans le centre historique de Berlin (Mitte), on observe un déplacement progressif vers l'ouest, vers les quartiers huppés de Charlottenburg, Schöneberg, puis les villas cossues de Grunewald ou de Wannsee. Ces lieux de vie contrastent avec le Scheunenviertel, largement mythifié dans l'imaginaire collectif. En effet, il n'y a jamais eu de ghetto au sens propre à Berlin ; la population qui le composait n'était pas majoritairement juive. En revanche, il est vrai qu'il concentrait de nombreux Juifs venus de l'Est, souvent très démunis, qui parlaient yiddish, étaient majoritairement orthodoxes et constituaient une population très visible avec leurs barbes, caftans, papillotes, kippot. On peut parler dans une certaine mesure d'un " shtetl » miniature où régnait une " subculture juive de l'Est », d'un quartier grouillant de petits commerces, de marchés, avec des rues emblématiques comme la Dragoner-, l'Artillerie- ou la Grenadierstraße ${ }^{23}$. Bien que ce milieu ne soit pas homogène (certains aspirent à l'ascension sociale, comme Mischket Liebermann, qui fuit à seize ans le « ghetto » orthodoxe de ses

21. Paul Lerner, The Consuming Temple: Jews, Department Stores, and the Consumer Revolution in Germany, 1880-1940, Ithaca / London, Cornell University Press, 2015.

22. Richarz in Lowenstein, Mendes-Flohr et al. (dir.), Umstrittene Integration, p. 62-63. Ces chiffres se rapportent à l'année 1907 : par comparaison, à cette date, $15 \%$ des avocats allemands étaient juifs et $6 \%$ des médecins.

23. Voir notamment l'étude de Martin Beradt, Die Straße der kleinen Ewigkeit, Berlin, Die Andere Bibliothek, 2001. Cette rue constituait le premier point d'attache et d'orientation pour les migrants arrivant à Berlin. 
parents et s'engage dans la lutte communiste tout en entamant une carrière d'actrice ${ }^{24}$ ), il modifie tout de même le profil social des Juifs berlinois ${ }^{25}$. La misère n'y est pas un mythe, comme le rappelle Joseph Roth en 1927 dans Juden auf Wanderschaft : «Kein Ostjude geht freiwillig nach Berlin ${ }^{26}$ ». Dans les années 1920, il devient synonyme des bas-fonds de Berlin, du monde de la petite criminalité.

\section{Épicentre religieux}

Au niveau religieux, Berlin joue incontestablement un rôle phare pour les autres communautés juives. C'est la plus grande d'Allemagne : en 1930, elle emploie 1500 personnes et dispose d'un budget de dix millions de Reichsmarks ${ }^{27}$. La poussée démographique rend nécessaire la construction de nouveaux lieux de prières. Pas moins de dix-sept synagogues sont érigées durant notre période : non pas la Nouvelle synagogue de l'Oranienburger Straße, qui est inaugurée en $1866^{28}$, mais celle de la Rykestraße (1904), de la Fasanenstraße (1912) - première synagogue de l'ouest de la ville -, celle de la Prinzregentenstraße (1930)... En 1932, Berlin compte au total quatre-vingt-quatorze synagogues et salles de prière, ce qui atteste une pratique religieuse encore très prononcée. De même, le nouveau cimetière de Weißensee, la " plus grande nécropole juive d'Europe », est ouvert en 1880, ainsi que de nombreuses institutions comme des foyers pour personnes âgées, un nouvel hôpital (1914), etc. ${ }^{29}$

Au-delà de cette dynamique, sa spécificité par rapport au reste de l'Allemagne réside dans son engagement majoritaire et particulièrement prononcé dans le courant libéral du judaïsme : il n'y a d'ailleurs qu'à Berlin qu'on le qualifie encore de "réformé », ce qui exprime sa radicalité ${ }^{30}$. Il faut rappeler que Berlin avait déjà joué un rôle central dans la naissance du mouvement de réforme juive à côté de Hambourg et Breslau. La Nouvelle synagogue de l'Oranienburger Straße était le centre flambant neuf de la réforme avec orgue, chœur et une nouvelle liturgie, le fameux « rite berlinois ", élaboré notamment par Louis Lewandowski (1821-1894), un des fondateurs de la musique synagogale moderne, par ailleurs le premier Juif admis à l'Académie des arts de Prusse. Abraham Geiger lui-même, arrivé à Berlin à la fin de sa vie en 1870, est impressionné par le climat particulièrement propice au judaïsme libéral qui y règne ${ }^{31}$.

24. Voir son autobiographie : Aus dem Ghetto in die Welt, Verlag der Nation, Berlin (Est), 1977.

25. On pense aussi à l'influence du yiddish sur le dialecte berlinois.

26. Joseph Roth, Juden auf Wanderschaft, Wien/ München, Christian Brandstätter Verlag, 2010.

27. Andreas Reinke, Geschichte der Juden in Deutschland 1781-1933, Darmstadt, Wissenschaftliche Buchgesellschaft, 2007, p. 121.

28. Au retour des troupes prussiennes victorieuses qui ont défait l'Autriche. Voir Laurence Guillon, «Leistung und Schicksal der Synagoge in der Oranienburger Strasse » (2013) sur le site La Clé des langues : [http://cle.ens-lyon.fr/allemand/leistung-und-schicksal-der-synagoge-in-deroranienburger-stra-e], dernière consultation le 8 avril 2019.

29. Voir Ulrich Eckhardt, Andreas Nachama, Jüdische Orte in Berlin, Berlin, Nicolai, 2005.

30. Voir Lowenstein, Umstrittene Integration, p. 101.

31. Lettre à son ami Bischofsheim en 1872, in Volker Spiess, Jüdisches Leben in Berlin, Berlin, Haude \& Spener, 1995, p. 8. 
Cela ne signifie pas, évidemment, que tous les Juifs de Berlin étaient libéraux, et le conflit avec la tendance néo-orthodoxe s'affirme en particulier avec la prise de distance de la communauté « séparée » Adass Jisroel, qui existait depuis 1869 et se voit reconnue comme personne morale en 1885. Elle regroupe environ $10 \%$ des membres, parmi les plus pauvres ou les plus récemment arrivés ${ }^{32}$. Cette communauté se dote d'institutions propres: synagogue, cimetière, et même un institut de formations des rabbins, le Rabbinerseminar d'Esriel Hildesheimer (1873), créé en réaction à l'ouverture de la Hochschule für die Wissenschaft des Judentums un an plus tôt. Elle devient l'institution de formation de rabbins orthodoxes la plus importante en Europe : Berlin joue donc aussi un rôle de premier plan dans le judaïsme orthodoxe, même s'il est minoritaire.

\section{Une créativité hors du commun}

Mais Berlin peut aussi être considéré comme un épicentre de la culture juive en dehors des institutions communautaires.

\section{Berlin, épicentre de la culture juive non religieuse}

Berlin est en effet le berceau de la science du judaïsme depuis la création du Verein für Cultur und Wissenschaft der Juden en 1819 par Leopold Zunz, Eduard Gans et Heinrich Heine. Plus tard, la ville joue un rôle phare dans son développement avec la Hochschule für die Wissenschaft des Judentums (séminaire de formation des rabbins et enseignants), dont le statut est privé car I'université refuse de reconnaître cette discipline en son sein. On y pratique la démographie, la sociologie, l'ethnologie appliquées aux Juifs. Cet établissement atteint son apogée en 1932 avec 155 étudiants inscrits. On notera la présence parmi eux d'une femme, Regina Jonas, qui rédige une thèse sur la possibilité pour une femme de devenir rabbin. Elle deviendra effectivement la première femme rabbin d'Allemagne, mais seulement en 1935. La République de Weimar est une époque particulièrement productive pour la science du judaïsme, avec cette fois deux pôles concurrents, Berlin et Francfort-sur-le-Main, où est créé le Freies Jüdisches Lehrhaus par Franz Rosenzweig en 1920, et où a enseigné Martin Buber. À Berlin, deux institutions voient le jour en 1919 : la Jüdische Volkshochschule et l'Akademie für die Wissenschaft des Judentums, l'une dédiée à la formation d'adultes intéressés par le judaïsme, mais pas nécessairement juifs, l'autre dédiée à la recherche ${ }^{33}$. L'Akademie est à l'origine une idée de Hermann Cohen, qui passe la fin de sa vie à Berlin et meurt en 1918, reprise par une trentaine d'intellectuels juifs qui se réunissent à Berlin début 1919 en plein chaos révolutionnaire (parmi eux Albert Einstein, Leo Baeck, Ernst Cassirer, Hermann Struck). La science du

32. Voir Ehrenfreund, Mémoire juive et nationalité allemande, p. 52.

33. Voir Sonia Goldblum, "Jüdisches Forschen oder jüdisches Lernen. Zwei jüdische Wissenseinrichtungen in Berlin in den 1920er Jahren » in Guillon, Knörzer (dir.), Berlin und die Juden, p. 89-108. 
judaïsme s'établit donc en dehors des séminaires rabbiniques, ce qui revêt un caractère particulièrement important car en 1918 encore, il n'existe pas de chaire d'études juives en Allemagne ${ }^{34}$.

Parallèlement au développement de ce réseau autour de la science du judaïsme, I'histoire juive étend elle aussi ses ramifications. Elle n'est plus uniquement l'affaire des théologiens et veut s'affirmer comme partie intégrante de l'histoire allemande. Ainsi, elle s'approprie les objectifs et les méthodes de I'historisme allemand $^{35}$. Pour Jacques Ehrenfreund, la communauté juive de Berlin est animée par une véritable passion de l'histoire, en interaction avec la nation allemande nouvellement constituée, et certainement liée à une crainte de voir un jour la culture juive disparaître. C'est en ce sens qu'il faut comprendre les efforts déployées pour créer des fonds d'archives et des collections de musées : en 1885 la Historische Kommission für die Geschichte der Juden in Deutschland « invente l'histoire judéo-allemande ${ }^{36}$ », et devra fermer en 1902, faute de moyens financiers. En 1905, c'est le Gesamtarchiv der deutschen Juden qui prend le relais (cette institution crée les premières archives juives au monde sous le titre de Germania Judaica), et finalement, la Gesellschaft zur Förderung der Wissenschaft des Judentums (1902) devient l'institution scientifique la plus active du judaïsme allemand. Il y a donc une volonté d'écrire l'histoire des Juifs dans le cadre national allemand, et l'idée (nouvelle) que la capitale du Reich doit centraliser les traces du passé des Juifs en Allemagne.

Enfin, Berlin s'affirme également comme la capitale de la «Renaissance juive ${ }^{37}$ », phénomène qui s'épanouit dans le sillage du sionisme et de la redécouverte d'influences culturelles venues de l'Est de l'Europe. Berlin devient ainsi le centre de la redécouverte de la culture yiddish. Le Scheunenviertel joue un rôle important de ce point de vue avec entre autres le théâtre juif créé en 1906 par Anton und Donat Herrnfeld, venus de Budapest en 1891, dans la Kommandantenstraße. Ces deux frères dirigeaient le théâtre, écrivaient les pièces et les jouaient euxmêmes ${ }^{38}$. Ce sont ensuite les auteurs d'expression hébraïque qui participent à ce renouveau juif, en faisant renaître la langue et la poésie hébraïques, comme Haïm Nahman Bialik (1873-1934), Samuel Joseph Agnon (1888-1970), Leah Goldberg (1911-1970), autour de lieux de sociabilité comme le «Café des Westens » ou le "Monopol ». Pour Fania Oz-Salzberger, cette rencontre de diverses influences culturelles juives à Berlin dans les années 1920 aurait été rien moins que le creuset de la culture israélienne actuelle ${ }^{39}$.

34. Les premières seront créées à Francfort, Cologne, Leipzig, mais Berlin n'est pas pionnier en ce domaine, notamment à cause de la réticence d'Adolf von Harnack, qui s'oppose catégoriquement à l'introduction des études juives au sein de la faculté de théologie.

35. Ehrenfreund, Mémoire juive et nationalité allemande, p. 130 et suiv.

36. Ibid., p. 1.

37. Voir à ce propos l'article de Delphine Bechtel dans le présent ouvrage, qui évoque l'article programmatique de Martin Buber en 1901.

38. Kurt Tucholsky les admirait beaucoup. Mais le théâtre ferme en 1916 ; il sera réutilisé par le Jüdischer Kulturbund, créé en juillet 1933.

39. Fania Oz-Salzberger, Israelis in Berlin, Frankfurt a. M., Jüdischer Verlag, 2001, p. 177. 


\section{Une surreprésentation dans le milieu académique}

D'autre part, Berlin est sans doute une des villes d'Europe où les Juifs réussissent le mieux dans le milieu académique. Comme le dit si bien Dominique Bourel, le prénom juif le plus répandu à Berlin sous la République de Weimar était sans doute "Herr Doktor ${ }^{40}$ ». En 1887, les Juifs représentaient $25 \%$ des élèves des lycées (Gymnasien). En 1907, $67 \%$ des enfants juifs poursuivaient leurs études après l'école primaire, contre $8 \%$ dans la population allemande globale.

Berlin est aussi la ville universitaire par excellence pour les étudiants juifs : ils constituent $17 \%$ des étudiants à Berlin en $1886-87^{41}$ et ne viennent pas seulement d'Allemagne, mais notamment de Russie (les Juifs de Russie parlant yiddish, l'allemand leur était accessible et les conditions d'études bien meilleures que dans leur pays). Cela est vrai notamment de quelques jeunes femmes, admises comme auditrices (sur autorisation explicite de tel ou tel professeur) à l'université de Berlin à partir de 1896, qui étudient souvent la médecine ${ }^{42}$. Marie Munk est la première femme à devenir avocate à Berlin en 1924 et juge en 1930, après avoir obtenu son doctorat en $1911^{43}$. Ces femmes constituent bien sûr une avantgarde et leur nombre reste restreint. Elles devront attendre 1908 pour obtenir des diplômes universitaires en Prusse.

Par ailleurs, les professeurs juifs sont de plus en plus nombreux, y compris dans les sciences humaines, alors qu'ils avaient jusqu'alors plus de chances de trouver un poste dans le domaine des sciences naturelles. La psychanalyse mérite également d'être mentionnée puisqu'en 1920 s'ouvre le Psychoanalytisches Lehrinstitut, qui compte de nombreux élèves de Freud et dont presque tous les étudiants sont juifs.

Enfin, on ne peut oublier les Prix Nobel juifs berlinois : Paul Ehrlich (médecine) en 1908, Fritz Haber (chimie) en 1918, Albert Einstein (physique) en 1921. Ce dernier est d'ailleurs moins connu comme violoniste de talent, qui a joué par exemple lors de concerts à la Nouvelle Synagogue avec le fils de Louis Lewandowski et incarne ainsi parfaitement la « Bildung » complète telle qu'on pouvait se la représenter à cette époque dans les milieux juifs cultivés.

\section{Au cœur de la vie intellectuelle et artistique}

La liste des artistes juifs ayant contribué au rayonnement de Berlin, au sens de « Mitgestalter», peut se lire comme un véritable «Who's who » de I'histoire culturelle de la ville. En 1871, Berlin n'est pas encore un centre culturel aussi réputé que Vienne ou Prague, mais il est en passe de le devenir. Force est de

40. Bourel in François, Westerholt (dir.), Berlin : Capitale, mythe, enjeu, p. 92.

41. Richarz in Lowenstein, Mendes-Flohr et al. (dir.), Umstrittene Integration, p. 58. (La proportion d'étudiants juifs dans l'Empire allemand est alors de $9 \%$.)

42. Voir Luise Hirsch, Vom Schtetl in den Hörsaal: jüdische Frauen und Kulturtransfers, Berlin, Metropol, 2010.

43. Monika Richarz, « Eine weibliche Avantgarde. Jüdische Frauen in Berlin als Studentinnen und Akademikerinnen », in Guillon, Knörzer, Berlin und die Juden, p. 180. 
constater une différence de générations entre l'époque de l'Empire, marquée par les valeurs de l'Anständigkeit, de la Bildung, et la République de Weimar, qui se caractérise par un style beaucoup plus libéré, même si cela est très schématique. En musique par exemple, Berlin apparaît comme le haut-lieu des tendances les plus novatrices, avec Arnold Schönberg, qui trouve à Berlin un public et une critique beaucoup plus réceptifs à son travail qu'à Vienne ${ }^{44}$ ou encore Kurt Weill, qui y écrit la musique de l'Opéra de quat'sous ${ }^{45}$. C'est également le cas des BeauxArts, notamment de l'impressionnisme, représenté par Max Liebermann ${ }^{46}$ et Lesser Ury, puis de l'expressionnisme (Ludwig Meidner, Jakob Steinhardt). L'image de la ville est également marquée dans les années 1920 par l'architecture d'Erich Mendelsohn, qui, outre la Tour Einstein à Potsdam, construit le grand magasin Schocken ainsi que des immeubles de bureaux résolument novateurs. Dans le monde du théâtre et du cinéma, les artistes juifs sont également légion. Évoquons bien sûr Max Reinhardt, qui s'installe à Berlin en 1894 et dirigera le Deutsches Theater à partir de 1905, et Ernst Lubitsch, fils d'un tailleur berlinois réputé, figure-clé des débuts du cinéma allemand avant son départ pour les États-Unis en 1923. II convient d'évoquer également un " art mineur » considéré comme typiquement berlinois, le cabaret, avec bien sûr Kurt Tucholsky, Walter Mehring, Fritz Grünbaum, Annemarie Haase...

En littérature, la liste est longue et il est impossible de procéder à une analyse de détail de toutes les œuvres liées intrinsèquement à Berlin. En tout cas, pour Marcel Reich-Ranicki, « l'élément berlinois a trouvé son reflet le plus net et le plus intense dans la prose d'auteurs juifs ${ }^{47}$ ". Évoquons bien sûr Alfred Döblin, qui a immortalisé le milieu de la petite criminalité dans son roman aux techniques novatrices, Berlin Alexanderplatz (1929), composé notamment à partir de différents articles qu'il avait écrits sur Berlin, et qui est resté son seul succès ${ }^{48}$. Franz Hessel publie la même année Spazieren in Berlin, qui remet au goût du jour la figure du flâneur, véritable "prêtre du genius loci » pour Walter Benjamin ${ }^{49}$. Beaucoup moins connu est Georg Hermann (1871-1944), le « Fontane juif », qui décrit la vie quotidienne berlinoise, en particulier à travers les sagas de familles

44. Laure Gauthier explique qu'avant tout, Berlin lui a laissé la possibilité de s'expliquer sur ses œuvres, même si celles-ci n'ont pas toujours été bien reçues. Voir « Arnold Schönbergs Aufenthalte in Berlin oder die Erfahrung des intensives Auslands » in Guillon, Knörzer, Berlin und die Juden, p. 137-162. Schönberg a séjourné trois fois à Berlin, de décembre 1901 à l'été 1903, puis de l'automne 1911 à 1915, et enfin de janvier 1925 à février 1933.

45. Kurt Weill vit à Berlin entre 1918 et 1933.

46. D'abord marginalisé en raison de son art non conventionnel, il deviendra président de l'Académie des Arts en 1920, puis citoyen d'honneur de Berlin en 1927.

47. Marcel Reich-Ranicki, Über Ruhestörer - Juden in der deutschen Literatur, München, DTV, 1993, p. 389-390.

48. La trajectoire de Döblin lui-même est caractéristique : après avoir grandi dans l'Est de la ville dans des conditions misérables, il parvient à ouvrir son premier cabinet de neurologue et psychiatre dans le quartier de Kreuzberg. Plus tard, il s'établira dans la Frankfurter Allee. Ce n'est qu'après le succès de Berlin Alexanderplatz qu'il s'installe sur le Kaiserdamm, où il vivra jusqu'à son départ en exil, le 28 février 1933. II ne reviendra pas à Berlin après 1945.

49. Cité par Changnam Lee, « Le flâneur urbain et la masse-nomade. Réflexion inspirée des textes de Benjamin et de Kracauer dans les années 1920-1930 », Sociétés 112 (2), 2011, p. 133. 
juives bourgeoises, Jettchen Gebert (1907) et Henriette Jacoby (1908), qui peuvent apparaître comme les reliques d'une idée de Bildung de plus en plus désuète dans l'Allemagne du XX $X^{\mathrm{e}}$ siècle. Hermann fut en son temps un auteur à succès, et ce pas seulement auprès du lectorat juif, mais il est tombé dans l'oubli après sa mort à Auschwitz ${ }^{50}$. Nous terminerons ce panorama nécessairement incomplet avec un dernier écrivain, Artur Landsberger (1876-1933), auteur d'un curieux roman " d'anticipation ", Berlin ohne Juden, qui paraît la même année que Mein Kampf, en 1925 : il s'agit d'une utopie négative, dans laquelle un parti antisémite prend le pouvoir et exclut progressivement les Juifs de la société allemande ${ }^{51}$. Mais à la différence de ce qui se passera plus tard dans la réalité, ils sont tous accueillis à bras ouverts par les nations étrangères, qui, en boycottant l'économie allemande, parviennent à ramener ses dirigeants à la réalité. Ce qui nous intéresse particulièrement dans le roman, c'est que la ville de Berlin, sans ses Juifs, est totalement paralysée : il y a pénurie d'avocats, de chauffeurs de taxis, la bourse s'effondre ; lorsqu'on veut séjourner au prestigieux hôtel Adlon, il faut apporter soi-même ses draps et on n'est autorisé à prendre un bain qu'une fois par semaine ; comble de l'absurde, on ne trouve pas un seul musicien capable de composer un hymne allemand. Comme le résume un Juif allemand ayant trouvé refuge à Paris : « Berlin est mort ${ }^{52}$ ». En 1933, Landsberger se suicide en voyant son scénario se réaliser.

Enfin, on ne peut passer sous silence le rôle éminent joué par des Juifs berlinois comme critiques littéraires (Alfred Kerr ${ }^{53}$, Julius Bab...), comme mécènes qui ont largement offert aux musées de Berlin les collections qui font leur renommée aujourd'hui encore (James Simon, Eduard Arnhold ${ }^{54}$...) ou encore tout simplement comme public - un public qui voyait en Fontane sa figure de proue ${ }^{55}$.

Cette inscription juive dans la culture allemande ou plutôt cette créativité exceptionnelle ont pu être interprétées comme un "âge d'or » de la relation judéo-berlinoise. Or ce phénomène a également sa part d'ombre : une haine à l'encontre des Juifs motivée par les théories raciales qui se développent à partir du milieu du XIXe siècle, bientôt appelée « antisémitisme ».

50. Voir notamment Godela Weiss-Sussex (dir.), Georg Hermann, deutsch-jüdischer Schriftsteller und Journalist, 1871-1943, Tübingen, Niemeyer, 2004.

51. Cette œuvre est probablement inspirée de Hugo Bettauer, Die Stadt ohne Juden, 1922, qui imagine un scénario similaire pour la ville de Vienne. II n'est cependant pas prouvé que Landsberger ait eu connaissance de ce roman.

52. Artur Landsberger, Berlin ohne Juden, Bonn, Weidle Verlag, 1998 [1925], p. 149.

53. Auteur de Berliner Briefe hebdomadaires publiées entre 1895 et 1900 dans le Breslauer Zeitung, qui dressent un panorama du Berlin de Guillaume II.

54. Voir l'article de Johanna Heinen dans le présent volume.

55. Comme le rappelle Theodor Fontane lui-même, non sans ironie, dans son poème publié en 1894 : «An meinem Fünfundsiebzigsten », in Joachim Krueger, Anita Golz (éds.), Gedichte, t. 2, Berlin, Aufbau, 1995, p. 466-467. 


\section{Une vie juive de plus en plus menacée}

Celui-ci marque la vie des Juifs indirectement, dans le sens où souvent, l'organisation de la « défense » était beaucoup plus thématisée au sein des familles juives que les traditions ou la religion, et que finalement, cet antisémitisme les a poussés à se redéfinir. Or, c'est précisément Berlin qui semble, une fois encore, en première ligne dans ce mouvement.

\section{Berceau du nouvel antisémitisme}

II ne s'agit pas de remonter au-delà du XIXe siècle, mais c'est déjà à Berlin qu'un des tout premiers professeurs de l'université, créée en 1810, Friedrich Rühs, s'applique à démontrer que les Juifs ne doivent pas avoir accès à la citoyenneté allemande tant qu'ils ne se convertissent pas : c'est en 1815, dans son pamphlet Über die Ansprüche der Juden auf das deutsche Bürgerrecht, qu'il développe sa théorie de l'État chrétien.

Le terme d'antisémitisme lui-même, ce néologisme, apparaît plus tard à Berlin sous la plume de Wilhelm Marr en 1879 dans un essai intitulé Der Sieg des Judenthums über das Germanenthum, avec le sous-titre évocateur Vom nichtkonfessionnellen Standpunkt betrachtet, et se répand très vite dans la langue parlée. Au même moment, le prédicateur de la cour impériale, Adolf Stoecker crée le Christlich-Soziale Arbeiterpartei, un parti sans précédent, qui mène un double combat contre les Juifs et les sociaux-démocrates. Il obtient un succès non négligeable jusqu'à la fin du siècle, puis il est victime du déclin de l'antisémitisme parlementaire. Troisième facteur concomitant, le célèbre professeur d'histoire Heinrich von Treitschke publie en novembre 1879 le pamphlet Ein Wort über unser Judentum, qui s'achève avec la tristement célèbre formule «Die Juden sind unser Unglück ${ }^{56}$ », promise à un long avenir. S'il ne croit pas qu'on puisse revenir sur les acquis de l'émancipation, il pense connaître les véritables raisons de la haine des Juifs qui, selon lui, se répand en Allemagne ${ }^{57}$ : I'arrogance des Juifs (Hochmut), qui refuseraient de s'intégrer et de devenir des Allemands, leur contrôle des médias et de l'opinion publique, et enfin, l'immigration en provenance de l'Est. Treitschke donne une caution intellectuelle de premier ordre à ce nouvel antisémitisme, cherchant à se démarquer d'un antijudaïsme violent et primitif tel qu'il avait pu s'exprimer au cours du XIX siècle, par exemple lors des émeutes « Hep Hep » en $1819^{58}$, et se met en scène comme un briseur de tabous. Son écrit a déclenché la

56. Preußische Jahrbücher 44, 1879, p. 572-575.

57. Il formule d'ailleurs soigneusement son essai de manière à apparaître comme un observateur extérieur, détaché de ce phénomène.

58. On désigne par cette formule des actions violentes contre les Juifs allemands, allant jusqu'au meurtre, parties de Wurtzbourg en août 1819, auxquelles assiste notamment le jeune Heinrich Heine à Düsseldorf. Le cri de ralliement « hep-hep » viendrait, selon certains historiens, de l'expression latine " Hierosolyma est perdita », ancien cri de guerre des croisés. 
« querelle de l'antisémitisme » (ainsi baptisée en 1965 59 ), qui reste un phénomène avant tout berlinois. Une « résolution des notables » est signée en novembre 1880 par 75 personnalités non-juives, dont le maire de Berlin, Max von Forckenbeck, pour dénoncer les propos de Treitschke. Les protestations de Juifs berlinois seront nombreuses (Ludwig Bamberger par exemple), celle de non Juifs beaucoup plus rares, mais l'intervention de Theodor Mommsen, Auch ein Wort über unser Judentum, où il qualifie l'antisémitisme de " tare congénitale du sentiment national ", est restée célèbre. Cette controverse a été suivie avec passion par l'opinion publique berlinoise. Pour finir, une pétition antisémite lancée en 1880 obtient 265000 signatures, mais elle est ignorée par le gouvernement, qui se contente de rappeler que la remise en cause de l'émancipation des Juifs n'est pas à l'ordre du jour. On remarque que durant la « querelle de l'antisémitisme », celuici se double bien souvent d'une haine de la grande ville, comme s'il s'agissait des deux faces de la même médaille - une argumentation que l'on retrouvera plus tard dans l'argumentaire nazi avec les images de la «Babel de la Spree » ou de la « Nouvelle Jérusalem ${ }^{60}$ ».

Ces trois événements se produisent exactement au même moment et convergent avec le "tournant conservateur » de Bismarck. Ils ont rendu l'antisémitisme « politiquement correct » et l'ont introduit dans différents milieux, où il se répand très vite, d'abord parmi les étudiants de Berlin ${ }^{61}$, puis dans plusieurs partis et courants politiques : au sein du parti conservateur, de nombreuses associations nouvelles, comme le Verein Deutscher Studenten, I'Alldeutscher Verband, le Bund der Landwirte... II devient ainsi un puissant mouvement idéologique et social à Berlin, relayé par d'autres intellectuels comme Eugen Dühring, autoproclamé socialiste, qui publie en 1881 Die Judenfrage als Racen-, Sitten- und Kulturfrage, et Adolf von Harnack, cité plus haut, qui publie en 1900 Das Wesen des Christentums. Cette idéologie antisémite est ainsi partie de Berlin pour devenir un ciment unificateur, un « code culturel » pour parler avec Shulamit Volkov ${ }^{62}$, qui rassemble les milieux conservateurs, nationalistes, hostiles à la modernisation, à l'industrialisation, mais aussi au féminisme, au développement des métropoles. Elle est symptomatique d'une jeune nation «nerveuse » qui se constitue en opposition à des « ennemis » présupposés : le catholicisme, la socialdémocratie, les Juifs...

\section{Réactions berlinoises}

Les réactions juives à l'antisémitisme s'organisent elles aussi en premier lieu à Berlin, qui devient l'épicentre de la défense (Abwehr) contre celui-ci. Tout

59. Avec la publication de l'ouvrage de Walter Boehlich (éd.), Der Berliner Antisemitismusstreit Frankfurt a. M., Inselverlag, 1965.

60. À propos des discours sur Berlin comme « bastion des Juifs », voir Schlör, Das Ich der Stadt, p. 226 et suiv.

61. Max Weber, alors étudiant à Berlin, observe une « jubilation frénétique » autour de Treitschke.

62. Shulamit Volkov, Antisemitismus als kultureller Code, München, Beck'sche Reihe, 2000. 
commence là encore avec les étudiants, exclus des corporations, qui créent en 1882 I'AkademischerVerein für jüdische Geschichte und Literatur. Celui-ci fusionne avec d'autres organisations pour former le Kartell-Convent deutscher Studenten jüdischen Glaubens en 1896. Parallèlement est créé le Verein zur Abwehr des Antisemitismus en 1891, qui a pour vocation de rassembler des chrétiens hostiles à ce mouvement de haine, mais qui compte aussi des Juifs. Puis ce sera la naissance du Centralverein deutscher Staatsbürger jüdischen Glaubens en 1893, institution phare des Juifs assimilés, qui symbolise le sentiment d'appartenance des Juifs à la nation allemande.

Berlin est également au cœur de la réaction que l'on qualifie généralement - en forçant le trait - d'« opposée » : le sionisme (ou les sionismes). La Berliner Zionistische Vereinigung est fondée par Heinrich Loewe dès 1897, un an après la parution de l'ouvrage de Theodor Herzl, Der Judenstaat, et même si le siège de la Zionistische Vereinigung für Deutschland s'établit d'abord à Cologne, il est transféré en 1905 à Berlin. L'Organisation sioniste mondiale y a également son siège à partir de 1911 sous la direction de Kurt Blumenfeld, ainsi que toutes les organisations affiliées : ZO, WIZO, KKL, KH ${ }^{63}$, Makkabi, Bar-Kochba... Pour Joachim Schlör, cette concentration des organisations sionistes à Berlin renvoie à un besoin fondamental de la grande ville pour les Juifs de la Diaspora : la ville apparaîtrait comme une compensation à la perte de leur terre ${ }^{64}$.

Le conflit entre sionistes et " assimilationnistes » envahit bientôt la vie communautaire berlinoise : le Jüdische Volkspartei est créé en 1919, rassemblant des sionistes, des orthodoxes et des Juifs de l'Est dans une alliance stratégique contre la majorité libérale. Ce parti dirigera la communauté juive de 1927 à 1931, sans induire cependant de changement révolutionnaire. Notons que très rares sont les Berlinois qui, malgré leurs convictions, mettent à exécution le projet du sionisme et réalisent leur aliyah : on peut évoquer Lotte Cohn, une des toutes premières femmes sionistes, architecte de formation, à émigrer en Palestine en 1921, puis bien sûr Gershom Scholem en 1923.

Une autre institution de poids sous la République de Weimar est fondée à Berlin en 1919 par Leo Löwenstein, le Reichsbund jüdischer Frontsoldaten : il milite pour la reconnaissance des soldats juifs tombés au front et plus généralement contre tout antisémitisme. II devient rapidement la deuxième organisation juive après le Centralverein en termes de membres, et dérivera vers une tendance nationalconservatrice dans les années 1930. À ce propos, Berlin est également le centre de mouvements juifs atypiques, caractérisés par un nationalisme allemand poussé à l'extrême, comme le Verband nationaldeutscher Juden fondé par un Berlinois, Max Naumann, qui reprochait au Centralverein ses positions trop mièvres (et militait notamment pour l'expulsion des Juifs de l'Est). La plupart de ses 3000 membres étaient Berlinois. De même, Hans-Joachim Schoeps fonde à Berlin son mouvement

63. Zionist Organization, Women's International Zionist Organization, Keren Kayemet Lelsrael, et Keren Hasseyod.

64. Schlör, Das Ich der Stadt, p. 267. Cette idée ne va pas d'ailleurs sans une certaine contradiction avec l'idéal sioniste du retour à la terre. 
Der deutsche Vortrupp en 1933, qui présente la particularité d'être favorable au national-socialisme à ses débuts. Faut-il y voir l'expression d'une haine de soi juive ${ }^{65}$ ? En tout cas, on ne peut s'empêcher d'y lire une sorte de schizophrénie : ces Juifs berlinois se voyaient comme tellement allemands qu'ils ne se sentaient pas eux-mêmes menacés en tant que Juifs par le national-socialisme ${ }^{66}$.

\section{Violences et exclusion}

Enfin, les Juifs sont victimes de la montée extrême de l'antisémitisme sous la République de Weimar, qui prend des formes particulièrement violentes à Berlin, où l'on voit réapparaître une forme d'antisémitisme primitif ("RadauAntisemitismus ») vis-à-vis duquel Treitschke avait justement pris ses distances. Ces actes de violence se manifestent de plus en plus souvent lors de manifestations culturelles : ainsi, en 1921, des émeutes à caractère antisémite ont lieu lors de la première de La Ronde de Schnitzler, perçue comme un œuvre « pornographique », ou encore en 1929, lors de la représentation de la pièce Le Marchand de Berlin de Walter Mehring, qui dut être retirée du programme. Mais on pense avant tout aux assassinats politiques qui ont secoué le régime : I'assassinat de Walther Rathenau le 24 juin 1922, mais également l'attentat contre Maximilian Harden ${ }^{67}$, la même année, qui décide de s'exiler en Suisse. Même la vie quotidienne des Juifs et des Juives n'échappe plus à cette insécurité : les 5 et 6 novembre 1923, ont lieu des pillages de magasins juifs dans le quartier des granges, motivés la prétendue «spéculation » des Juifs de l'Est, sur fond de crise économique. La police n'intervient pas et la population juive est défendue par les forces du Reichsbund jüdischer Frontsoldaten. On compte un mort parmi les assaillants. Au printemps 1927, puis à l'automne 1930, des agressions se produisent sur le Kurfürstendamm (rebaptisé « Kohnfürstendamm » dans la presse national-socialiste ${ }^{68}$ ) à l'encontre de passants « d'apparence juive », qui sont roués de coups. En septembre 1931, ces violences se répètent, orchestrées par la SA, à l'occasion du Nouvel An juif.

La violence et l'exclusion se traduisent également sur le plan économique. Christoph Kreutzmüller a montré par exemple que si les Juifs possédaient 20 à $25 \%$ des commerces de la ville au début des années 1930, cela ne signifiait nullement que les commerces juifs avaient une force économique particulière, encore moins une toute-puissance : au contraire, cela était plutôt le signe d'une pénurie économique, car il s'agissait de petits, voire de minuscules commerces durement touchés par l'inflation puis par la crise de 192969. Par leur structure professionnelle, les Juifs ont en effet été beaucoup plus concernés que le reste

65. Voir l'article de Martine Benoit dans le présent volume.

66. Sur ces tendances nationalistes, voir notamment Reinke, Geschichte der Juden, p. 127-128.

67. Considéré comme " l'enfant terrible » de l'Empire, il mène campagne contre l'empereur Guillaume II dans sa revue Die Zukunft. II était par ailleurs lié à Walther Rathenau, qui publie son essai Höre, Israel! dans la revue de son ami en 1897.

68. Voir Schlör, Das Ich der Stadt, p. 238.

69. Christoph Kreutzmüller, "Le marchand de Berlin. Les commerces juifs à Berlin, 1918-1942 » in Guillon, Knörzer (dir.), Berlin et les Juifs, p. 157-161. 
de la population par ces deux phénomènes : on observe de nombreuses faillites et fermetures de magasins dès les années 1920, bien avant les actions de boycott organisées par le NSDAP. Ce n'est pas un hasard si la toute première caisse d'emprunt juive («Darlehenskasse ») est créée à Berlin en 1924. En 1930, un quart des Juifs de Berlin sont dépendants des aides sociales de la communauté (soupes populaires, foyers, travail...). Dans ces conditions, les boycotts allaient bientôt rendre cette lutte contre l'exclusion totalement vaine.

\section{Conclusion}

Nous pouvons donc conclure à une ambiguïté fondamentale de la période étudiée: les vies juives à Berlin oscillent constamment entre intégration, épanouissement culturel et antisémitisme, qui apparaissent comme les deux faces d'une même médaille. Elles sont caractéristiques $d^{\prime}$ '« une communauté à la vitalité, à la créativité et à l'inventivité peu communes [...] à la fois très diversifiée et foncièrement solidaire qui est portée de l'avant par le dynamisme de l'Allemagne unifiée, par celui de sa capitale en pleine croissance et plus encore par son dynamisme propre $^{70}$ ", et qui sera bientôt victime de nouvelles formes de haine. Tout comme les entrepreneurs qui ont donné à Berlin une aura internationale, les hommes politiques qui ont marqué de leur sceau une certaine culture du libéralisme et de la tolérance, une pléiade d'acteurs culturels, dont on ne connaissait pas, la plupart du temps, les origines juives, ont fait la réputation de Berlin, en particulier dans les années 1920. II est impressionnant de constater qu'en 1933, malgré l'arrivée au pouvoir de Hitler, Berlin est encore le théâtre de nombreuses actions culturelles, dont le symbole tragique est sans doute l'ouverture du musée juif de Berlin, à côté de la synagogue de l'Oranienburger Straße, le 24 janvier 1933. Le Kulturbund deutscher Juden créé en juillet 1933 rassemblera quant à lui des initiatives d'une étonnante vitalité. Tout ce foisonnement intellectuel motivera le retour de nombreux émigrés après 1945, comme l'actrice Valeska Gert, qui, à la question de savoir pourquoi elle était revenue au « pays des meurtriers », répond: "Ich brauchte ein Band zu Berlin ${ }^{71 " . ~ A u j o u r d ' h u i ~ e n c o r e, ~ l ' œ u v r e ~ c u l t u r e l l e ~ d e s ~}$ Juifs à Berlin fait l'actualité des médias : ainsi, la Galerie James Simon vient d'être inaugurée sur l'île aux Musées en hommage au mécène qui a offert aux musées de Berlin le fameux buste de Néfertiti, l'un des hommes les plus riches de Prusse, qui a consacré sa fortune à la culture et à la bienfaisance dans sa ville natale.

70. Étienne François, « Préface », in Ehrenfreund, Mémoire juive et nationalité allemande, p. XI-XII.

71. Anat Feinberg, Wieder im Rampenlicht? Jüdische Rückkehrer in deutschen Theatern nach 1945, Göttingen, Wallstein, 2018, p. 39. 


\section{ANNEXE}

\section{Quelques personnalités juives berlinoises}

Nées à Berlin :

Leo Arons (1860-1919), physicien et homme politique (SPD). Privatdozent à I'université de Berlin à partir de 1890, son poste fut menacé en raison de son appartenance politique - c'est d'après lui qu'a été nommée la "Lex Arons » (1898) interdisant aux membres du SPD d'enseigner dans les universités prussiennes.

Julius Bab (1880-1955), dramaturge et critique de théâtre. Co-fondateur du Kulturbund Deutscher Juden en 1933.

Herbert Baum (1912-1942), électricien, ouvrier de l'entreprise Siemens, dirigeant communiste d'un groupe de résistance, exécuté en 1942.

Walter Benjamin (1892-1940), philosophe, critique littéraire, traducteur et écrivain, auteur d'une œuvre très diversifiée, notamment d'une autobiographie : Berliner Kindheit um die Jahrhundertwende, écrite durant l'exil et publiée à titre posthume notamment grâce à Gershom Scholem.

Eduard Bernstein (1850-1932), homme politique (SPD), exilé en Suisse en 1878, puis en Angleterre, co-auteur du programme d'Erfurt (1891), militant pour la réformisme au sein de la social-démocratie.

Gerson von Bleichröder (1822-1893), banquier, conseiller financier de Bismarck, anobli en 1872 (premier Juif non converti à l'être).

Curt Bois (1901-1991), acteur, scénariste et réalisateur, représentant du cinéma muet. Exil en 1934.

Lotte Cohn (1893-1983), architecte, une des premières femmes diplômées de la Technische Hochschule Charlottenburg, émigration en Israël en 1921.

Arthur Eloesser (1870-1938), historien de la littérature, écrivain et journaliste (critique de théâtre pour le Vossische Zeitung).

Valeska Gert (1892-1978), actrice, danseuse et humoriste, ouvre un cabaret à Berlin en 1932. Exil aux États-Unis.

Moritz Goldstein (1880-1977), journaliste (notamment pour le Vossische Zeitung) et écrivain, célèbre pour son article « Der deutsch-jüdische Parnass » en 1912, qui suscite un grand débat sur la « symbiose judéo-allemande ».

Maximilian Harden (Felix Ernst Witkowski, 1861-1927), journaliste et polémiste, éditeur du journal Die Zukunft très critique vis-à-vis de Guillaume II. Ami de Walther Rathenau, il est lui aussi victime d'une attaque antisémite en 1922, suite à laquelle il se réfugie en Suisse.

Georg Hermann (Georg Borchardt, 1871-1943), journaliste, critique d'art et écrivain. Exil en 1933.

Adolf Jandorf (1870-1932), entrepreneur, fondateur du Kaufhaus des Westens en 1907. 
Regina Jonas (1902-1944), diplômée de la Hochschule für die Wissenschaft des Judentums, première femme rabbin en 1935, déportée et assassinée à Auschwitz.

Gertrud Kolmar (Gertrud Chodziesner, 1894-1943), traductrice, préceptrice, poétesse.

Artur Landsberger (1876-1933), critique littéraire, romancier à succès, auteur de Berlin ohne Juden en 1925, suicide en 1933.

Julius Lessing (1843-1908), historien de l'art, premier directeur du musée des Arts décoratifs (Kunstgewerbemuseum) de Berlin à partir de 1872.

Max Liebermann (1847-1935), peintre, co-fondateur de la Berliner Sezession, directeur de l'Académie prussienne des Arts de 1920 à 1933.

Ernst Lubitsch (1892-1947), fils du tailleur réputé, Simon Lubitsch, il est engagé comme acteur par Max Reinhardt au Deutsches Theater, puis pour le cinéma. Après 1918, il réalise lui-même ses propres films, puis émigre aux États-Unis en 1923.

Walter Mehring (1896-1981), journaliste et écrivain satirique, collaborateur de la Weltbühne, auteur de chansons de cabaret. Auteur de Der Kaufmann von Berlin en 1929.

Marie Munk (1885-1978), première femme avocate et juge (1924), exil aux ÉtatsUnis.

Erich Mühsam (1878-1934), écrivain, anarchiste, fondateur de la Ligue socialiste en 1908 avec Gustav Landauer et Martin Buber, participant actif de la révolution de 1918. Arrêté après l'incendie du Reichstag, il est assassiné au camp d'Oranienburg-Sachsenhausen.

Max Naumann (1875-1939), avocat, fondateur du Verband nationaldeutscher Juden en 1921.

Rudolf Nelson (Lewysohn, 1878-1960), compositeurs de chansons à succès, de musiques de films et d'opérettes, ouvre son cabaret sur le Kurfürstendamm en 1920, exil en 1933.

Hugo Preuß, (1860-1925), juriste, professeur de droit à l'université de Berlin, homme politique (DDP), considéré comme le père de la constitution de Weimar, ministre de l'Intérieur en 1919.

Emil Rathenau (1838-1915), ingénieur, fondateur de la Deutsche Elekrizitätsgesellschaft en 1883 (plus tard Allgemeine Elektrizitätsgesellschaft, AEG).

Walther Rathenau (1867-1922), industriel, écrivain et homme politique (DDP), ministre de la Reconstruction en 1921, puis des Affaires étrangères en 1922, assassiné le 24 juin 1922.

Alice Salomon (1872-1948), militante féministe, thèse en 1906 sur les causes de l'inégalité salariale entre hommes et femmes, crée la Soziale Frauenschule en 1908, convertie en 1914.

Erich Salomon (1886-1944), photographe et journaliste, considéré comme le pionnier du photoreportage (employé par la maison d'édition Ullstein). 
Hans-Joachim Schoeps (1909-1980), historien, fondateur en 1933 de l'association Der deutsche Vortrupp. Gefolgschaft deutscher Juden, favorable au nationalsocialisme à ses débuts.

Gershom Scholem (1897-1982), historien et philosophe, spécialiste de la kabbale et de la mystique juive. Émigration en Palestine en 1923. Auteur d'une autobiographie : De Berlin à Jérusalem - Souvenirs de jeunesse (1984).

Ernst Simon (1899-1988), philosophe et historien, engagé volontaire en 1916, gravement blessé à Verdun. Études à Berlin, puis collaborateur de Buber au Freies Jüdisches Lehrhaus (Francfort/M.). Sioniste, émigration en 1928 en Palestine mandataire.

James Henry Simon (1851-1932), homme d'affaires, mécène, à l'origine de prestigieuses collections des musées de Berlin (buste de Néfertiti notamment).

Kurt Tucholsky (1890-1935), journaliste et écrivain, satiriste, converti en 1918. Exil en Suède à partir de 1930.

Jakob van Hoddis (Hans Davidsohn, 1887-1942), poète expressionniste, précurseur du dadaïsme, célèbre pour le poème «Weltende » publié en 1911.

Theodor Wolff (1868-1943), écrivain et journaliste, rédacteur du Berliner Tageblatt de 1906 à 1933, membre fondateur du DDP.

Ayant vécu à Berlin durant une période significative :

Ludwig Bamberger (1823-1899), homme politique (libéral de gauche), instigateur du système monétaire unitaire allemand.

Leo Baeck (1873-1956), appelé à Berlin en 1912, rabbin, enseignant à la Hochschule für die Wissenschaft des Judentums.

Elisabeth Bergner (Elisabeth Ettel, 1897-1986), actrice, engagée par Max Reinhardt en 1923. Exil en Angleterre.

Kurt Blumenfeld (1884-1963), sioniste, à Berlin pour ses études en 1904, secrétaire de la Zionistische Vereinigung für Deutschland en 1909, secrétaire général de l'Organisation Sioniste Mondiale de 1911 à 1914, rédacteur de la revue sioniste Die Welt, président de la Zionistische Vereinigung de 1924 à 1933. Exil en Palestine.

Oscar Cassel (1849-1923), juriste, conseiller municipal (libéral de gauche), premier Juif à obtenir le titre de citoyen d'honneur de Berlin en 1914. Président du Verband deutscher Juden en 1917.

Paul Cassirer (1871-1926), critique et marchand d'art, qui a joué un rôle important dans la reconnaissance de la Berliner Sezession et de l'impressionnisme.

Hermann Cohen (1842-1918), philosophe, passe sa retraite à Berlin pour se consacrer à la religion juive, a enseigné à la Hochschule für die Wissenschaft des Judentums.

Paul Dessau (1894- 1979), compositeur et chef d'orchestre, musique d'avantgarde notamment pour le cinéma. Exil en France, puis aux États-Unis.

Ernst Deutsch (1890-1969), acteur, engagé au Deutsches Theater par Max Reinhardt en 1917. Exil aux États-Unis. 
Alfred Döblin (1878-1957), neurologue, psychiatre, auteur de Berlin Alexanderplatz en 1929, enfance à Berlin, émigration en France en 1933, puis aux États-Unis. Converti en 1941.

Tilla Durieux (Ottilie Godefroy, 1880-1971), actrice, appelée à travailler à Berlin avec Max Reinhardt et Erwin Piscator, représentante du cinéma muet dans les années 1920. Épouse le peintre Eugen Spiro en 1904, puis le marchand d'art Paul Cassirer en 1910, qui se suicide après leur divorce en 1926. Exil en Suisse en 1933.

Paul Ehrlich (1854-1915), professeur à la faculté de médecine de Berlin à partir de 1887, prix Nobel en 1908 pour ses travaux sur l'immunité.

Albert Einstein (1879-1955), physicien, nommé directeur du Kaiser-WilhelmInstitut für Physik par l'Académie des Sciences et des Lettres de Prusse à Berlin en 1914. Exil en 1933.

Hanns Eisler (1898-1962), compositeur et théoricien de la musique. Élève de Schönberg, il s'installe à Berlin en 1925, collaborateur de Brecht à partir de 1925. Exil en France, puis aux États-Unis.

Lion Feuchtwanger (1884-1958), critique de théâtre, metteur en scène et dramaturge, auteur de Jud Süß en 1925 (nouvelle pervertie dans le film de Veit Harlan), qui lui vaut une renommée internationale. Ami et collaborateur de Brecht. Vit à Berlin entre 1930 et 1932. Exil en 1933.

Samuel Fischer (1859-1934), arrivé en 1881 à Berlin, il fonde la maison d'édition Fischer en 1886.

Karl Emil Franzos (1848-1904), écrivain, réside de 1887 à 1904 à Berlin.

Ludwig Geiger (1848-1920), fils d'Abraham Geiger (1810-1874), à Berlin depuis 1870. Privatdozent en histoire à I'université de Berlin, auteur d'une Histoire des Juifs de Berlin en 1871.

Levin Goldschmidt (1829-1897), juriste et homme politique (libéral de gauche), conseiller financier de Guillaume $\mathrm{I}^{\mathrm{er}}$.

Alexander Granach (Jessaja Granach, 1890-1945), acteur, enfance dans le quartier des granges ; carrière au Theater am Nollendorfplatz sous la direction d'Erwin Piscator, vedette des débuts du cinéma muet (Nosferatu en 1922).

Sammy Gronemann (1875-1952), avocat, écrivain, sioniste, arrivé à Berlin en 1905, il étudie au Séminaire rabbinique d'Esriel Hildesheimer, co-fondateur du Schutzverband deutscher Schriftsteller. Exil à Paris, puis en Palestine.

Hugo Heimann (1859-1950), éditeur, mécène et homme politique (SPD), conseiller municipal, citoyen d'honneur de Berlin en 1926.

Max Hermann-Neiße, journaliste et poète, à Berlin de 1917 à 1933.

Franz Hessel (1880-1941), écrivain et traducteur, auteur de Heimliches Berlin en 1927 et de Spazieren in Berlin en 1929.

Esriel Hildesheimer (1820-1899), rabbin de la communauté orthodoxe Adass Jisroel à partir de 1869, créateur du Séminaire rabbinique en 1873.

Magnus Hirschfeld (1868-1935), médecin, fondateur de l'Institut de sexologie à Berlin, militant contre la persécution des personnes homosexuelles. 
Friedrich Hollaender (1896-1976), compositeur (de musiques de films comme celle de L'Ange bleu) et réalisateur, études à Berlin, collaborateur de Max Reinhardt, directeur de cabaret dans les années 1920. Exil en 1933.

Mascha Kaléko (1907-1975), poétesse et écrivaine, représentante de la Nouvelle Objectivité. Exil aux États-Unis, s'installe en Israël en 1960.

Berthold Kempinski (1845-1910), négociant en vin, restaurateur, arrivé à Berlin en 1872.

Alfred Kerr (1867-1948), critique littéraire, auteur de nombreux articles sur Berlin. Exil en 1933.

Otto Klemperer (1885-1973), études de musique à Berlin, notamment auprès de Schönberg, chef d'orchestre à l'opéra Kroll. Converti au catholicisme, il émigre aux États-Unis.

Eduard Lasker (1829-1884), juriste et homme politique (libéral de gauche), instigateur de l'« Austrittsgesetz » (billet de sécession) en 1876.

Else Lasker-Schüler (Elisabeth, 1869-1945), dessinatrice et poétesse, s'installe à Berlin en 1894 après son mariage. Exil en 1933.

Louis Lewandowski (1821-1894), arrivé en 1834 à Berlin, musicien, créateur de la liturgie réformée.

Mischket Liebermann (1905-1981), arrivée en 1914 à Berlin (Scheunenviertel), militante communiste, devient actrice, engagement au Deutsches Theater.

Isidor Loewe (1848-1910), industriel, employé dans la fabrique d'armes de son frère.

Ludwig Loewe (1837-1886), frère d'Isidor, entrepreneur, fondateur en 1869 d'une fabrique de machines à coudre, plus tard «Waffen- und Munitionsfabriken Berlins ", secrétaire particulier de Lassalle.

Peter Lorre (1904-1964), acteur à Berlin à partir de la fin des années 1920, révélé par son rôle dans M le maudit de Fritz Lang en 1931. Exil en 1933.

Rosa Luxemburg (1871-1919), femme politique (KPD), arrivée à Berlin en 1905, assassinée le 15 janvier 1919 lors de la « semaine sanglante».

Erich Mendelsohn (1887-1953), architecte, après avoir suivi une partie de ses études à Berlin, il y ouvre un cabinet d'architecte en 1918. Connu pour ses réalisations architecturales comme la Tour Einstein à Potsdam (1920), ainsi que de nombreux immeubles de bureaux à Berlin, représentatifs de l'expressionnisme. Exil en 1933.

Lina Morgenstern (1830-1909), écrivain, militante pour les droits des femmes, instigatrice des cuisines populaires (d'où son surnom « Suppenlina »).

Rudolf Mosse (1843-1920), éditeur (Berliner Tageblatt, Berliner Morgenzeitung, Berliner Volkszeitung...).

Emil Orlik (1870 -1932), peintre, professeur à la Kunstgewerbeschule à Berlin à partir de 1905.

Max Pallenberg (1877- 1934), acteur, à partir de 1914 au Deutsches Theater de Berlin. Epouse Fritzi Massary en 1917. 
Jakob Plessner (1871-1936), sculpteur (nombreux bustes de Moïse Mendelssohn, Oskar Tietz...).

Eva Reichmann (1897-1998), historienne et sociologue, représentante du Centralverein à partir de 1924 avec son mari Hans Reichmann.

Max Reinhardt (Max Goldmann, 1873-1943), acteur, metteur en scène et intendant de théâtre, à Berlin à partir de 1894, dirige le Deutsches Theater à partir de 1905.

Julius Rodenberg (Julius Levy, 1831-1914), journaliste et écrivain, à partir de 1861 à Berlin, auteur de Bilder aus dem Berliner Leben (1885).

Joseph Roth (1894-1939), écrivain et journaliste, observateur de la vie berlinoise dans les années 1920, auteur notamment de Juden auf Wanderschaft en 1927.

Arthur Ruppin (1876-1943), économiste et sociologue, sioniste, études et doctorat à Berlin, directeur du centre statistique et démographique juif de 1902 à 1907, puis envoyé en Palestine pour étudier les possibilités d'installation sioniste.

Arnold Schönberg (1874-1951), pionnier de la musique dodécaphonique, a vécu à Berlin au cours de trois longs séjours (1901-1903, 1911-1915 et 1925-1933), élu à l'Académie des Arts en 1925. Converti en 1898, il récuse cette conversion en 1933.

Anna Seghers (Netty Reiling, puis Radvanyi, 1900-1983), écrivaine, installée à Berlin en 1925, elle quitte la communauté juive, membre du KPD à partir de 1928. Exil en 1933, d'abord en France puis au Mexique.

Kurt Singer (1885-1944), neurologue et musicien, chef d'orchestre. Études à Berlin, fondateur du chœur des médecins en 1912, intendant du Städtische Oper en 1927, directeur du Kulturbund à partir de 1933.

Hermann Tietz (1837-1907) et son neveu Oskar (1895-1975), directeurs de grands magasins, premier grand magasin ouvert dans la Leipziger Straße en 1900 ; plus grande enseigne de grands magasins d'Europe en 1926.

Leopold Ullstein (1826-1899), éditeur, fondateur des éditions Ullstein en 1887 (Berliner Zeitung, Berliner Morgenpost, Berliner Illustrierte Zeitung...).

Lesser Ury (1861-1931), peintre, représentant de l'impressionnisme, membre de la Berliner Sezession, depuis 1887 à Berlin.

Kurt Weill (1900-1950), musicien, compositeur, à Berlin entre 1918 et 1933, collaborateur de Brecht (musique de L'opéra de quat'sous). Exil en France, puis aux États-Unis.

Robert Weltsch (1891-1982), journaliste, éditeur de la Jüdische Rundschau à Berlin (1919-1938), émigration en Palestine en 1938.

Georg Wertheim (1857-1939), s'installe à Berlin en 1885, où il ouvre plusieurs grands magasins. Converti en 1906.

Leopold Zunz (1794-1886), co-fondateur de la science du judaïsme.

Arnold Zweig (1887-1968), écrivain, pacifiste et communiste. Installé à Berlin dans les années 1920, il y écrit Der Streit um den Sergeanten Grischa (1928), ainsi que Das ostjüdische Antlitz (1922). 